Décadrages Décadrages

cınéma, à travers champs Cinéma, à travers champs

3 | 2004

Hitchcock côté cour

\title{
Une visite au Louvre de Jean-Marie Straub et Danièle Huillet : « un abîme où l'œil s'enfonce... »
}

\section{François Albera}

\section{OpenEdition}

\section{Journals}

Édition électronique

URL : http://journals.openedition.org/decadrages/558

DOI : $10.4000 /$ decadrages.558

ISSN : 2297-5977

Éditeur

Association Décadrages

Édition imprimée

Date de publication : 10 avril 2004

Pagination : 74-84

ISBN : 978-2-9700582-0-5

ISSN : 2235-7823

Référence électronique

François Albera, « Une visite au Louvre de Jean-Marie Straub et Danièle Huillet : « un abîme où l'œil s'enfonce... » », Décadrages [En ligne], 3 | 2004, mis en ligne le 29 avril 2013, consulté le 01 mai 2019. URL : http://journals.openedition.org/decadrages/558; DOI : 10.4000/decadrages.558 


\section{Une visite au Louvre de Jean-Marie Straub et Danièle Huillet :}

"un abîme où l'œil s'enfonce..."

par François Albera

1 Joachim Gasquet, Cézanne, Bernheim jeune, Paris, 1921 (rééd. régulières depuis 1926, notamment Cynara, Dijon, 1988).
Rarement un film aussi court (48 minutes) et aussi simple d'apparence (une sculpture et quatorze tableaux filmés frontalement, fixement, entièrement, avec un commentaire off) aura déclenché des ondes de choc d'aussi longues portées. Tant par son paradoxe (un film où la part des cinéastes semble congrue puisque l'on compte trois plans «de cinéma" seulement, mais qui apparaît comme l'un de leurs plus personnels sinon le plus intime) que par le foisonnement de sa matière qui ne cesse de développer des niveaux ou des chemins d'une projection à l'autre, voire tout simplement dès lors qu'on y réfléchit quelque peu.

Ce film est bref on l'a dit et apparemment simple: sur un texte de Joachim Gasquet qui rapporte des propos de Cézanneㄹ, on montre successivement une sculpture (la Victoire de Samothrace) et quatorze tableaux (la Source d'Ingres, [l'Assassinat de] Marat de David, la Remise des Aigles de David, les Noces de Cana de Véronèse, la Fille de Jä̈re de Véronèse, Concert champêtre de Titien, la Cuisine des anges de Murillo, le Paradis de Tintoret, le Triomphe d'Homère d'Ingres, Femmes d'Alger [dans leur appartement] de Delacroix, l'Entrée des croisés [à Constantinople] de Delacroix, le Naufrage [le Radeau de la Méduse] de Géricault, le Grand Sous-bois du combat de cerfs [Rut du printemps ou Combat de cerfs] de Courbet, l'Enterrement à Ornans de Courbet). Les tableaux sont filmés entièrement, avec leur cadre, il n'y a qu'à trois reprises cadrage d'une partie seulement d'un tableau (un seul, les Noces de Cana, à deux reprises, et un second, le Paradis de Tintoret, une seule fois), on passe d'un tableau à l'autre le plus souvent par une coupe franche, quatre fois un écran noir les sépare.

Pourtant la brièveté se révèle d'emblée complexe puisque l'exploitation en salle d'Une visite au Louvre offre le film "en miroir», deux fois de suite dans deux versions légèrement différentes (la seconde de 47 minutes). La simplicité, quant à elle, se complique plus vite encore: 
d'abord ce commentaire de Cézanne - qui nous parvient par la médiation d'un témoin, Joachim Gasquet, qui a transcrit en les synthétisant les propos du peintre quinze ans après sa mort et bien après les avoir entendus (il le rencontre en 1895). Gasquet avait lui-même des velléités d'écrivain et a sans doute récrit - il en convient - les propos de Cézanne et certaines formules frappantes lui doivent ${ }^{2}$. Mais outre qu'à comparer ces "Conversations» avec la correspondance du peintre, ou d'autres témoignages ${ }^{3}$, on retrouve la même tonalité, on voit bien que ces propos appartiennent à un même courant de pensée qui a des échos peu après chez Rilke $\mathbf{4}$ et encore plus tard chez Elie Faure par exemple 5 . Enfin, ce commentaire du peintre est dit par une voix féminine s'exclamant dans ses derniers mots: "Je suis Cézanne!», dissociant de la sorte le genre (grammatical) du sexe. La narratrice, Julie Koltai, adopte une surprenante diction faite de rage contenue et de fragilité, de souffle et de césures qui rythme le texte en blocs prosodiques aux arêtes parfois coupantes, parfois douces, délivrés de manière proche du rap.

Il y a donc une série de décalages, de couches historiques, sociales, culturelles et psychologiques qui étagent ce texte que les cinéastes ont recomposé, ôtant ici un mot, là un passage entier, interpolant une partie dans une autre, omettant toute la part narrative, et presque tout ce qui relève du dialogue (les questions et déclarations de Gasquet) pour en faire un monologue seulement interrompu trois fois par de brèves questions énoncées par Jean-Marie Straub lui-même. En un sens, Une visite au Louvre se rapproche ainsi de Chronik der Anna Magdalena Bach.

Le nombre de tableaux est limité à quatorze, mais le commentaire en convoque quelques autres; certains sont nommés (le Déjeuner sur l'herbe de Manet opposé au Déjeuner champêtre du Titien), d'autres suggérés à travers un nom de peintre (un portrait de Tintoret "que Manet a copié») ou une œuvre (Giotto - «je ne connais pas»-, Cimabue, L'Angelico - dont les «maladresses» et les "naïvetés " ne l'intéressent pas -, Uccello - les perspectives -, Holbein, Clouet, Raphaël - maîtres de la ligne -, Michel Ange - dont Tintoret disait avoir voulu allier le dessin avec la couleur du Titien -, Vélasquez - à propos du chien de l'Enterrement -, Renoir - "cet enfant de chœur, ce rouge joufflu... Renoir peut y venir...»-, Corot - «il y a plus de lumière chaude [dans un intérieur de Delacroix] que dans tous ces paysages de Corot»), ou par allusion (Gauguin: «ils cernent brutalement leurs bonshommes, leurs objets, d'un trait brut, schématique, appuyé, et en teintes plates on remplit jusqu'au bord»), il est enfin des tableaux implicites (comme la Source de Courbet qui s'oppose à celle d'Ingres ici décriée). Puis tout se complique encore dans la logique de succession des œuvres et ce qui les relie ou les
2 Cézanne lui écrit: "vous qui voulez être philosophe..." (30 avril 1896) (Paul Cézanne, Correspondance [éd. par John Rewald], Grasset, Paris, 1937, p. 222).

3 Paul Cézanne, Correspondance, op. cit., Ambroise Vollard, Paul Cézanne (1914), Emile Bernard, Souvenirs sur Paul Cézanne, (1921), etc.

4 Rainer Maria Rilke, Lettres sur Cézanne, Seuil, Paris, 1991 [1907].

5 Elie Faure, Histoire de l'art, Le Livre de Poche, Paris, 1976 (5 vol.) [1909-1926]. 
6 Voir Décadrages $\mathrm{n}^{\circ}$ 1-2, automne 2003, p. $148-160$. oppose, logique qui s'enrichit évidemment à la deuxième vision. Des thèmes plastiques, des motifs se mettent à circuler d'un tableau à l'autre (le pied par exemple, le chien, la cruche, l'eau, le sang...).

Le film est encadré par deux plans d'extérieur: le premier montre, sur sa longueur, le bâtiment du Louvre depuis le Pont du Carrousel de droite à gauche et retour, s'arrêtant dans l'axe du pont que parcourent des passants, des véhicules tandis qu'au loin, sous les voûtes, il y a l'entrée du musée. Le dernier plan est un mouvement panoramique sur un sous-bois, qui suit une source parmi les fougères, les buissons et les feuillages, les troncs que çà et là des taches de soleil illuminent. Au milieu du film, un troisième plan d'extérieur, la Seine bordée d'une rangée de platanes dont les feuilles sont violemment agitées par le vent, vue depuis le Louvre, en plongée, silencieux.

La structure du film nous fait ainsi passer des abords du musée - dans un environnement urbain et peuplé - à un espace rural, inhabité, au terme d'un parcours qui est la «visite» (c'est-à-dire de la suite de tableaux commentés que n'interrompt qu'un aperçu sur l'extérieur).

Or le scénario initial6 prévoyait d'achever le film sur un mouvement panoramique inverse du premier qui eût balayé le bâtiment une seconde fois et serait allé cadrer les tours de Notre-Dame. Le changement opéré au montage est donc considérable: en reprenant un plan qui ouvrait un film antérieur - Operai, contadini -, les cinéastes ont effectué un geste décisif: le parcours de la visite est vectorisé à partir de l'opposition extérieur-intérieur-extérieur, le troisième temps sortant complètement de l'espace annoncé par le titre et le début du film, celui du Louvre, à Paris, et de sa visite. L'opération est d'une grande violence, elle indique une sortie, une fuite, un dehors que le plan de la Seine (image fixe mais mobilité de la rivière et entrées et sorties de champ des bateaux qui se succèdent: une péniche, un bateau-mouche, une vedette rapide) suggérait mais qu'il donnait presque comme interdit, empêché (enfermement).

Qu'est-ce que cette aspiration à laquelle le film appelle et qu'il accomplit? Une sortie dans la nature que les tableaux tentent chacun à leur manière, dont Cézanne juge, lui qui allait inlassablement "au motif» sur la Sainte-Victoire et en mourut, qu'elle est le critère de la peinture. Ce plan final, c'est, enfin, "l'entrée lyrique de la nature, l'odeur des feuilles mouillées, les parois moussues de la forêt, le murmure des pluies, l'ombre des bois, la marche du soleil sous les arbres...", mais accomplie au-delà de la peinture: dans le cinéma, dans la saisie de l'image et des sons d'un lieu complexe, touffu, bruissant et chantant, animé de mille mouvements minuscules, à la fois tapi et promis à l'épanouissement 
dans la lumière qui s'aperçoit dans des trouées. On peut en effet parler de dépassement et d'au-delà de la peinture car ce plan panoramique circulaire (dû à Renato Berta) s'instaure au terme d'un trajet dans la peinture qui a abouti à une limite : le trou béant du cimetière d'Ornans, un abîme. Et c'est au moment où la voix proclame: "Je suis Cézanne!» qu'il surgit. La proposition est donc assertive : il n'y a pas dans ce film de tableau de Cézanne, il y aura un plan de cinéma, un plan straubien? 7 .

On pourrait méditer sur ce coup de force du film qui s'est imposé sans doute aux cinéastes après l'éprouvant tournage dans les musées - et le plus souvent leurs réserves -, après le regard incarcéré vers l'extérieur, depuis le musée. En même temps ce coup de force est des plus cézanniens: le peintre, s'adressant à Emile Bernard, en 1904 et 1905, oppose à plusieurs reprises le Louvre à la nature: "Le Louvre est un bon livre à consulter, mais ce ne doit être encore qu'un intermédiaire. L'étude réelle et prodigieuse à entreprendre, c'est la diversité du tableau de la nature.» (12 mai 1904); "Le Louvre est le livre où nous apprenons à lire. Nous ne devons cependant pas nous contenter de retenir les belles formules de nos illustres devanciers. Sortons-en pour étudier la belle nature...» (1905) 8 .

Avant cette issue, qu'on pourrait presque dire fatale pour la peinture, l'opposition intérieur-extérieur se joue tout au long du film dans le choix des tableaux. Leurs sujets, leurs genres: paysage, scènes de plein air ou au contraire scène d'intérieur. Tableau «sur le motif» ou d'atelier. Non sans contradictions: les Femmes d'Alger, dit le commentaire, sont plus dans la lumière que les paysages de Corot ou les scènes de bataille... D’ailleurs, passée la Victoire de Samothrace - sur laquelle on reviendra: Sainte-Victoire inaugurale du film -, on commence avec la Source d'Ingres et on aboutit à l'Enterrement à Ornans. Du chiqué ingresque ("le rocher de carton n'échange rien de son humidité pierreuse avec le marbre de cette chair mouillée... ou qui devrait l'être»), on aboutit à "l'entrée lyrique de la nature» de Courbet. Mais cette apothéose, équivalent au XIX $^{\mathrm{e}}$ siècle du Paradis du Tintoret, est saisie en deux toiles tragiques : un combat de cerfs où toute la sensualité du sous-bois est aussi promesse de souffrance et de mort, et surtout la procession funèbre de l'Enterrement encadrée, clôturée par les falaises de la Roche du Mont et de la Roche Founièche qui dominent le cimetière, lamento monumental qui ouvre un gouffre sous les yeux et les pas du spectateur: la fosse obscure aux bords desquels la foule se presse comme le visiteur depuis l'espace qu'il occupe ${ }^{9}$. Ce vide, cette béance attire et menace de tout engloutir. "L'abîme où l'œil s'enfonce» des Noces de Cana n'est plus ici un «état de grâce coloré», un bonheur, le miracle ou la floraison des vignes de
7 Comme dans Cézanne (1989) un passage de Der Tod des Empedokles (1987) poursuivait les plans de la Sainte-Victoire filmés sur place.

8 Paul Cézanne, Correspondance, op. cit., Lettres CLXVIII et CLXXXIII, p. 261 et 275.

9 Rappelons que cette "machine" qu'est l'Enterrement à Ornans présente des personnages en pied et en grandeur réelle (et même un peu plus, ils avoisinent 2 mètres), que Courbet y détourne un format réservé à la peinture d'histoire pour évoquer un événement trivial et accorder ainsi à des gens ordinaires (certains, à l'époque, ont dit "des gueux") un statut réservé aux grands hommes ou aux personnages représentatifs de l'ordre social au pouvoir (dans le film, la confrontation - à distance - de cet Enterrement et de la Remise des Aigles ou du Triomphe d'Homère est significative). Si le Musée d'Orsay ne rapetissait pas cette toile en l'exposant mal, sans doute sa puissance apparaîtrait-elle mieux aux visiteurs. Mais de l'occultation qu'elle subissait du temps de Cézanne à son amoindrissement aujourd'hui, il faut certainement voir une logique de l'institution muséale. 
10 C'est I'hypothèse pour partie hasardeuse de Michael Fried dans le Réalisme de Courbet, Gallimard, Paris, 1993 [1990], chap. 3.

11 Sans compter celle que Courbet a peinte à l'encontre de la "suave" Source désincarnée d'Ingres, où une femme bien en chair tourne le dos au spectateur et laisse l'eau asperger son corps, qui fait un avec la roche, l'eau jaillissante et les branches tandis qu'à ses pieds un reflet étonnamment statique dédouble ses chevilles.
Palestine à la naissance du Sauveur. C'est un trou noir. C'est pourquoi quand retentit le cri: "Je suis Cézanne!", correspondant, dans le livre de Gasquet, au moment où le peintre, en larmes, en fureur, vitupérant les gardiens qui veulent fermer, quitte le Louvre comme un fou, cette expulsion, cet écroulement des murs du musée basculent dans un plan straubien de sous-bois où l'on entend l'eau d'une source et les chants d'oiseaux, avant que, dans le noir cette fois, s'élève le chant d'un choral de Jean-Sébastien Bach. Loin du Louvre, dans cette nature appelée sans cesse par Cézanne et après un tableau qui tient son organisation, le mouvement sinueux de sa procession en direction de la fosse, du son de la Loue qu'en cet endroit on ne peut manquer d'entendre et qui organise la disposition des personnages ${ }^{\mathbf{1 0}}$, les cinéastes délivrent ou accueillent le proscrit Cézanne.

Entre ces deux peintures, ces deux "sources» en somme (l'une que l'on ne fait qu'«entendre»11), Cézanne a exprimé et cité des tableaux qu'il a envisagés sous l'angle de la physique des sensations, de l'air qui circule, de la matérialité des couleurs, du mouvement dans les formes en les rapportant toujours à un effet de réel.

Dans son commentaire des Noces de Cana, il définit un protocole très précis :

«Fermez les yeux... ne pensez plus à rien. Ouvrez-les! On ne perçoit qu'une grande ondulation colorée, une irisation, une chaleur harmonieuse, un abîme où l'œil s'enfonce, une sourde germination... on naît au monde vrai, on devient soi-même... Il faut d'abord perdre conscience, descendre aux racines sombres, enchevêtrées des choses, en remonter avec les couleurs, s'épanouir à la lumière avec elles, savoir voir, sentir..."

Etonnante proximité avec le cheminement préconisé par Husserl et plus tard Merleau-Ponty du "retour aux choses mêmes", un monde d'avant la connaissance abstraite, intellectuelle, scolastique. Ce qu'on appelle la "réduction phénoménologique». Mais ce protocole cézannien concerne la vision de l'art, d'une peinture, non de la réalité. Ici la peinture est proposée comme une médiation permettant l'appréhension vraie des choses à condition que le peintre sache faire passer dans sa matière et sa facture - son ouvrage - la logique du monde réel.

Dans le protocole évoqué ci-dessus, on a délibérément omis ce qui concernait la peinture, rétablissons-le:

«Fermez les yeux... ne pensez plus à rien. Ouvrez-les! On ne perçoit qu'une grande ondulation colorée, une irisation des couleurs, une richesse des couleurs. C'est ça que doit nous donner d'abord le tableau, une chaleur harmonieuse, un abîme où l'œil s'enfonce, une 
sourde germination... Tous ces tons vous coulent dans le sang... on se sent ravigoté. On naît au monde vrai. On devient soi-même. On devient de la peinture... Pour aimer un tableau, il faut d'abord l'avoir bu ainsi, à longs traits. Perdre conscience. Descendre avec le peintre aux racines sombres, enchevêtrées, des choses, en remonter avec les couleurs, s'épanouir à la lumière avec elles, savoir voir, sentir...»

La peinture offre donc un "guide» au spectateur. S'il se met lui-même en état de disponibilité, suspend son savoir (entendement), se laisse surprendre par ce qui apparaît sur la toile (non l'apparence de celle-ci - ce qu'elle figure -, mais son apparaître), il pourra refaire ce chemin de sensation qu'a effectué le peintre. Dans un passage non retenu dans le film, Cézanne rapporte que Courbet, un jour, avait peint ce qu'il avait devant lui sans l'avoir identifié et qu'il reconnut après-coup un fagot de bois, une fois les couleurs sur sa toile. C'est très exactement cette disponibilité, cette "virginité du regard»que recherchait Cézanne ${ }^{\mathbf{1 2}}$, une disposition dans laquelle il apprit à se mettre puisque toute une partie de sa carrière s'est déroulée dans la copie des maitres, dans le travail d'atelier, avant qu'il ne découvre le plein air et ne se convainque qu'il ne pouvait plus faire autre chose. Il reste que ce double protocole engage deux niveaux de réalité, celui du peintre devant la nature puis celui du spectateur devant le tableau, et que le «retour aux choses mêmes » via un tableau suppose soit que l'on vise les choses réelles à travers les enchevêtrements de lignes ou les arrangements de couleurs, au-delà d'eux (c'est la position de Husserl ou de Sartre), soit que le tableau participe de cette réalité dans son être même. C'est cette seconde solution qu'adopte manifestement Cézanne.

Ce qui, dans son propos, garantit cette médiation de l'art par rapport aux choses, c'est le métier, la pratique matérielle du peintre, ses instruments, les substances. Au long de son commentaire Cézanne insiste sur un aspect de cette pratique: les fonds. Les Vénitiens, les grands peintres avaient un métier, ils savaient peindre des fonds: les dessous. "Une immense grisaille", une "première emprise» sur laquelle venaient ensuite les couleurs et les glacis. On trouve cela chez Delacroix - sauf quand il est trahi par les «droguistes» - : l'Entrée des Croisés a pâli parce que "les fonds [se sont] éteints». On le trouve chez Courbet. Celui-ci, "c'est la force, le génie qu'il mettait par-dessous». Mais les modernes ont perdu ce savoir-faire et dès lors leurs tableaux flottent. Il y manque quelque chose, un frisson (Manet). Ce qui distingue les peintres modernes de ces telluriques, c'est qu'ils peignent par morceaux, ils manquent le tout. Ils font des images non de la peinture.
12 " ... je continue à chercher l'expression de ces sensations confuses que nous apportons en naissant" écrit-il à Joachim Gasquet (lettre CXXIXbis, s. d., Correspondance, op. cit., p. 227). 
13 On entend sourdement le texte pavesien des Dialoghi con Leuco et La Luna e i falò que travaillait Dalla Nuba alla resistanza (1978).

14 Outre de nombreux articles dans la Revue du cinéma des années 1946-1948 et l'Ecran français des mêmes années, voir Henri Lemaître, Beaux-Arts et cinéma, Cerf, Paris, 1956 et André Bazin, "Peinture et cinéma", Qu'est-ce que le cinéma?, Cerf, Paris, 1985 [1958].
Le métier de surcroît ne suffit pas: David, Ingres sont peut-être les derniers à savoir leur métier, mais ils le sacrifient à un système, à l'idée abstraite, l'idéal et du coup en bazardent la puissance d'être. La peinture comme matière, c'est le lien au réel, à la terre, à la roche ${ }^{13}$. La "première emprise» de Véronèse, cette "vaste grisaille» est "comme un morceau de la terre avant que le jour, l'esprit se lève». Ainsi, du fond, on touche aux "racines sombres, enchevêtrées des choses » et on peut remonter jusqu'à la lumière ensuite, s'épanouir, connaître l'extase. Sinon, resté en surface, le tableau est guetté par l'idéal sans corps, le décoratif. L'exemple "pied idéal» est à cet égard probant: repéré chez David (où dans la Remise des Aigles, l'un des officiers est immobilisé en pleine course, le pied droit, botté, levé vers l'arrière qui se détache avec préciosité sur la culotte blanche de son voisin), il est ramené au sol dans le Murillo (la Cuisine des anges), en opposition à la lévitation du saint "jaunâtre" avant d'être exemplifié dans le Paradis de Tintoret où sa "figure» procède du fond, des dessous.

\section{La force meurtrière de l'art}

Filmer la peinture, filmer les tableaux... La question surgit après la Deuxième Guerre mondiale. Est-ce l'effet, différé d'une quinzaine d'années, des réflexions de Valéry (l'ubiquité) et de Benjamin sur la reproduction des œuvres d'art et son action en retour? Celui, plus proche, des écrits de Malraux - qui exploite les précédents à sa manière - dans les Voix du Silence? La conviction de ce dernier est que la reproduction des œuvres dans les albums, les livres crée un "musée imaginaire» qui permet un certain type d'appropriation, l'agrandissement des tailles, la comparaison, etc., pratiques déjà courantes dans le siècle (ainsi Wölfflin), mais que Malraux systématise en s'adressant au grand public. Quoi qu'il en soit, le cinéma fait son affaire de cette question qui mobilise Bazin et quelques autres à partir des films de Luciano Emmer puis d'Alain Resnais, succédant à plusieurs tentatives des années trente signées Henri Storck, Auguste Baron, Paul Heilbronner, André Cauvin 14. Le cinéma s'empare de la peinture, dépasse la statique photographie, fût-elle organisée en série de détails et jouât-elle des éclairages "révélateurs». Le cinéma «trahit» la peinture et par là même il la sert ou même la sauve, dit Bazin, coutumier du procédé rhétorique du renversement: "c'est en dénaturant l'œuvre, en brisant ses cadres, en s'attaquant à son essence même que le film la contraint à révéler certaines de ses virtualités secrètes». En quoi consiste cette dénaturation salvatrice? A «[dissoudre] l'œuvre picturale dans la perception naturelle, en sorte que nulle culture, nulle initiation n'est requise pour jouir immédiatement 
et l'on pourrait même dire, par force, de la peinture imposée à l'esprit par les structures de l'image cinématographique, comme un phénomène naturel».

L'argument, on le voit, pourrait sembler rejoindre un certain propos cézannien, cette continuité que le peintre recherchait entre la peinture et la réalité. Mais la différence éclate cependant: pour Bazin il n'est pas question de dessous, de racines enchevêtrées, ni même de "réduction phénoménologique» («Fermez les yeux, ne pensez à rien, ouvrez-les!»), car il n'est pas question de matière ni de matériau, substrat, lieu de "germination" d'où surgira l'œuvre, mais de perception optique immédiate, d'apparence, il n'est pas question de peinture mais d'image: une «œuvre au second degré» naturalisant la peinture.

Cinquante ans plus tard cette formule s'est banalisée à tel point que les films sur l'art, "palettes» et autres documents plus ou moins didactiques qu'aiment à présenter les musées (il y eut les enchaînements de diapositives en fondus ou en simultané, les films, les émissions télé) dans leurs expositions ou vendre en cassettes vidéo puis CD Rom, puis DVD dans leurs boutiques ont, comme le disait fort bien Malraux, intellectualisél'art. Encore ne faut-il pas entendre par là «rendu intelligible» ou cosa mentale, mais dépouillé de toute matérialité, de toute substance, de toute référentialité avec l'objet-tableau, l'objet-sculpture. Il a sans doute été utile de répandre par la photographie - comme on le fit jadis par la gravure - la connaissance de l'art mondial dans le public, mais la démarche s'est sinon inversée du moins fortement altérée depuis Malraux et Skira. Aujourd'hui toutes ces médiations éloignent les visiteurs des œuvres: toujours, ils finissent par préférer aux originaux les diapos ou les films dont les images sont plus flatteuses (luminosité, transparence), et maintenant les reproductions numériques si scintillantes et qu'on manipule à l'envi (zoom!). Même s'ils se rendent pourtant sur place en foule, ils n'en demeurent pas moins captés par les écrans... Malraux balançait, dans le Musée imaginaire, entre un engouement pour la reproduction, la circulation des œuvres et, quand même, leur sacralisation (le Surnaturel, l'Intemporel...). C'est tout l'enjeu de sa «religion de l'art» qu'il veut substituer à l'art religieux. Le constat hegelien l'effraie. Sa conviction nécessite de garder les rituels et les temples. Aussi, conclut-il quelque part, le musée imaginaire ramène au Louvre, à l'original15.

Au-delà de ces atermoiements, tourner le dos à cette inflation, cette dilution est un geste violemment salutaire car c'est un geste critique : c'est celui que font Jean-Marie Straub et Danièle Huillet.

Or s'appliquer à filmer le plus soigneusement possible, en $35 \mathrm{~mm}$, en mesurant la lumière et la température de couleur afin qu'il n'y ait
15 "... un intellectualisme sans précédent dans l'art... sa destruction des appartenances" (p. 220); "L'ensemble des ouvrages consacrés à l'art ne reproduit pas un musée qui n'existe pas: il le suggère - et plus rigoureument, le constitue... II crée un lieu imaginaire qui n'existe que par lui." (p. 231); "Mais ce domaine qui fait du plus vaste Louvre une île, ramène à tous les Louvres ses fidèles, qui sont les leurs. Parce que les disques n'ont pas détruit les concerts; parce que nous voulons retrouver la perfection particulière ou l'irremplaçable grain de peau, l'âme réelle ou imaginaire qui n'appartient qu'à l'original..." (p. 231) 
16 Malraux, le Musée imaginaire, Gallimard "idées-Art", Paris, 1965 (édition remaniée et complétée), p. 175, ill. 126 "La Victoire de Samothrace" [photo de la statue cadrée serrée, incomplète, sur fond noir, tournée vers la gauche] (auparavant dans les Voix du silence, Gallimard, Paris, 1951, la même Victoire tronquée sur fond noir, p. 84); p. 235, photo de la Victoire (ill. 170) vue d'en dessous, sur un promontoire, ailes déployées sur un fond de mur atténué, ombré (dans l'édition de 1951, p. 67, le fond est gris uni). aucune disparité d'un plan à l'autre, en filmant à basse vitesse (6 images seconde dans certains cas) afin de suppléer un éclairage insuffisant, en choisissant soigneusement de centrer l'objectif au plan du tableau, dussent-ils pour cela ériger un échafaudage de 6 mètres de haut, en s'en tenant à des plans fixes qui durent, cadrés entièrement, y compris le cadre du tableau, etc., c'est s'affronter à de multiples difficultés. La difficulté d'accéder à l'objet plutôt qu'à ses succédanés.

S'efforcer de respecter les bords du tableaux, son cadre, son plan, c'est-à-dire s'interdire de le "dissoudre», justement, dans la "perception naturelle", c'est précisément permettre que se révèlent ses "virtualités secrètes». La Fille de Jaïre de Véronèse, petit tableau dont le format est décidément sans rapport avec celui de l'écran, devait fatalement laisser voir une surface importante du mur où il est accroché. Or le tableau comporte une voûte ouvrant sur un lointain dont le côté gauche est hors-cadre. Placer le tableau au bord gauche de l'écran eût fait redoubler cette coupure du cadre pictural par le cadre cinématographique et créé un effet de hors-champ. C'est à quoi se refusent les cinéastes qui placent le tableau à droite et ainsi laissent au spectateur voir cette coupure du cadre et refusent de la confondre avec celle de l'écran.

La portée considérable de ce film sous cet aspect de la «reproduction", on peut la vérifier aisément en confrontant une quelconque reproduction photographique d'une des quatorze toiles - avec le film; l'une des multiples effigies de la Victoire de Samothrace dans un catalogue, un livre d'art, en carte postale vendue au Louvre et les deux plans qui la montrent dans ce film. Prenons le cas "non-quelconque» de son apparition dans les livres de Malraux. Dans les Voix du Silence, puis dans le Musée imaginaire, la Métamorphose des dieux et dans le Surnaturel - qui sont autant de variations... Elle apparaît dans deux versions, choisies manifestement par l'auteur et liées à son propos. Lors de sa première apparition, la Victoire est sur un fond noir qui l'aplatit sur la page et dans un cadrage rapproché qui la détaille en excluant les jambes et une partie des ailes. Le commentaire de Malraux est alors assez vague. Quand la statue revient, plus loin, c'est en pied cette fois (et en ailes), en contreplongée, avec le socle-promontoir où elle est disposée. Le commentaire porte précisément sur la mise en scène qu'en fait le Louvre. C'est une allégorie, la figure du destin sur sa proue, etc. C'est aussi bien la Silver Lady, l'effigie de la calandre de Rolls-Royce que s'était amusé à analyser Panofsky 16 ...

Regardons maintenant le film des Straub. Il y a également deux vues de la sculpture. Pour commencer la statue apparaît dans son espace. Il $\mathrm{y}$ a derrière elle le mur et ses pierres ajointées, il y a le lieu où elle prend 
place, il y a «l'air» qui circule autour d'elle. Le cadrage laisse un espace au-dessus de la tête manquante. L'éclairage ne fonde aucune dramaturgie de l'ombre et de la lumière - procédés pour indexer le volume, le relief. Le "piqué» de la prise de vue (due à William Lubtchansky) est tel que le détail du marbre, les plis de la tunique collée aux jambes, le grain de la pierre soient perceptibles. Quand la parole démarre en disant "Tenez regardez-moi ça...", cela fait quelques secondes qu'on la regarde déjà intensément puisqu'elle impose sa force, son dynamisme, sa matérialité qui s'inscrivent dans le cadrage et l'angle et la lumière choisis. L'injonction de la parole décuple cet effet sensible et le porte plus loin: "c'est tout un peuple, un moment héroïque dans la vie d'un peuple, mais les étoffes collent, les ailes battent, les seins se gonflent..."

Et puis ces mots terribles: "Quand la tête s'est détachée, allez, le marbre a saigné...»

Cette parole, non seulement pulvérise toute appréhension allégorique, toute abstraction - cette abstraction que retient Malraux penché sur ses photos inertes -, elle introduit violemment la chair, le sang, le crime peut-être...

Carl Einstein parle quelque part de «la force meurtrière de l'œuvre d'art»17.

Quand retentissent les paroles de Cézanne: "Je n'ai pas besoin de voir la tête pour imaginer le regard, parce que tout le sang qui fouette, circule, chante dans les jambes, les hanches, tout le corps, il a passé en torrent dans le cerveau, il est monté au cœur. Il est en mouvement, il est le mouvement de toute la femme, de toute la statue, de toute la Grèce..." on reconnaît ainsi formulé ce qu'on voyait grâce au cadrage, à l'angle, à la durée du plan : l'espace où la tête fait défaut au plan figuratif est chargé de sa présence, elle ne manque pas dans le mouvement du corps. Il n'y a là nul goût du fragment, de la ruine ni même transmutation dans ce «monde de l'art» où l'on peut subsister sans tête, sans bras, sans être pour autant incomplet, car ce monde-de-l'art ne fait pas circuler de sang dans ses marbres et ses toiles! Or c'est de cela que parle Cézanne... Là encore comparons avec Malraux qui parle du caractère incomplet mais accompli par là même de la statue puisque la voilà parmi l'intemporel de l'art, avec les ébauches de Michel-Ange, avec Rodin 18...

A quoi nous mène l'intellectualisme de Malraux? A dire - cela l'obsède - que retiré de son appartenance (au milieu qui l'a vu naître, lui a assigné une place), l'art ne peut renaître que dans le monde de l'art. "Pour le chrétien Cézanne, écrit-il, un crucifix roman n'est pas le Christ mais une sculpture» $19 .$.
17 Carl Einstein, "Notes sur le cubisme", Documents, $n^{\circ} 3$, juin 1929, p. 152.

18 Commentaire en note: "La mutilation de la Victoire de Samothrace nous révèle, de façon immédiate et péremptoire, ce " qui aurait dû être " l'invention de l'ange parfait - et d'ailleurs celle de la Victoire. Or cet ange, cette Victoire imaginaires, les arts qui figurèrent les anges et les Victoires ne les eussent ni conçus ni acceptés. La Victoire de Samothrace n'est pas tout à fait une invention humaine. Nous pouvons l'imaginer intacte, et la supposer admirable, mais elle change de nature. La vraie surgit hors du temps, plus proche des ébauches de Michel-Ange et du Balzac de Rodin que d'aucune statue grecque; et l'absence de tête qui lui donne un mouvement sans analogue dans la sculpture antique, depuis le bas de la draperie jusqu'à la pointe des ailes, confirme cette intemporalité à quoi elle doit d'être si souvent représentée comme symbole de l'art. Les conservateurs du Louvre l'ont bien compris qui ont exposé isolé, et non mêlé aux œuvres hellénistiques, le chef d'œuvre du Destin." (p. 90).

19 Malraux, Le Musée imaginaire, op. cit. (édition remaniée et complétée), p. 179-180. 
20 Elie Faure, que bien des accents placent dans le même espace de pensée et de sensibilité que Cézanne dans son Histoire de l'art, écrit que les Impressionnistes aidèrent celui-ci à "secou[er] les influences qui le tyrannisaient...: Courbet, puis Daumier, puis Delacroix, puis, bien qu'on l'ait moins vu, Corot, puis, en remontant plus haut sur la trace des âmes, Rubens, Véronèse, Michel-Ange. II leur fut redevable de la liberté qu'il conquit péniblement et lentement sur la séduction despotique des grands œuvres, de considérer les héros de la peinture non comme des guides qu'on a le devoir de suivre, mais comme des témoins qu'on a le droit d'invoquer" (l'Art moderne, 2, op. cit., p. 158-159). C'est bien autre chose que dit Cézanne à Gasquet...
Or voilà aussi ce que ce film, son texte et ses images déplacent: Cézanne ne se préoccupe peut-être pas de chrétienté et d'agenouillement, mais il n'en projette pas pour autant l'art dans sa sphère autonome, il cherche à en trouver la force, non la seule forme. Et cette force est dans la proximité avec la Nature, peut-être oserions-nous dire, en citant Georges Bataille de manière déplacée, «jusque dans l'approbation de la mort».

Aux amateurs - devenus légions avec la banalisation des propositions de Warburg - de "rapprochements", le film des Straub comme les commentaires cézanniens offrent un enchaînement qui n'a rien de futile ni de seulement formel: de ce marbre qui saigne quand la tête est tombée à la fosse d'Ornans, via l'assassinat de Marat ou les combats de cerfs, il ne cesse d'être question de sang et d'eau : l'eau qui reste de l'eau claire (Ingres "parbleu, n'a pas de sang») et l'eau changée en vin des Noces de Cana, changée en sang.

Cette visite au Louvre a, pour Cézanne, la forme d'un "manifeste». D'ailleurs elle s'achève sur une esclandre. Il ameute le monde, veut alerter les journaux, Vallès, il crie "Qu'on foute le feu au Louvre... si on a peur de ce qui est beau». Le mot d'ordre aura son heure de gloire quelques années plus tard. Il y a là une "politique» cézanienne face à la société mais aussi face à ses contemporains, ceux qui croient bon rompre avec les classiques pour faire du nouveau et qui ne savent pas rendre hommage au monde. C'est une charge contre le refus de l'héritage, du passé20.

"Quand on ne sait pas, dit Cézanne, on croit que ceux qui savent vous obstruent. Alors qu'au contraire, si on les fréquente, au lieu de vous encombrer, ils vous prennent par la main, et vous font gentiment, à côté d'eux, balbutier votre petite histoire».

Il resterait à évaluer ce que Jean-Marie Straub et Danièle Huillet, qui avaient faite leur la charge de Schönberg contre le "modernisme» et Stravinsky "le petit modernski», entendent dire du monde du cinéma en reprenant ce manifeste cézannien, de ceux qui, comme David, "pens[ent] à ce qu'on dirait du peintre» et non de Marat.

Mais ceci est une autre histoire... 